\title{
Effectiveness of Web-Based Tutorials: Performance on Statistical Concepts
}

\author{
Swati Nandkishor Nere, Eugenia Fernandez, Charles Feldhaus, Jill Goodwin \\ Purdue School of Engineering \& Technology, Indiana University Purdue University Indianapolis \\ Indianapolis, United States
}

\begin{abstract}
This study was designed to examine the effectiveness of Web Based Tutorials (WBTs) and the correlation between students' self-efficacy score for self-regulated learning and their learning performance using WBTs. Participants were graduate students $(N=$ 14) enrolled in a statistics course during a single semester. The results of this study showed that WBTs were effective for learning statistics concepts. However, there was no correlation between students' self efficacy score for self regulated learning and their learning performance using WBTs. Additional investigation showed that the classroom instruction mode was more effective than the WBT.
\end{abstract}

Keywords: $\quad$ Self-efficacy for self regulated learning, web-based tutorials, learning styles.

\section{INTRODUCTION}

Web-based tutorials (WBTs) have become an important and integral part of distance education [1]. Distance education is a rapidly growing medium that is used in almost every field for training and education. Convenience, learning at one's own pace, and around-the-clock online accessibility are some of the possible reasons for its growing popularity.

Effective use of WBTs and multimedia can increase student learning [2]-[5] and help students to comprehend complex concepts that sometimes are difficult to understand in a face-to-face class. Computer-based demonstrations and tutorials may prove beneficial to students' learning in a course.

In educational literature, self-regulation studies are often referred to as Self-Efficacy for SelfRegulated Learning (SESRL, henceforth referred as SRL). SRL is a comprehensive construct that focuses on students' performance and achievement of learning processes in educational settings by focusing on how students motivate, plan, monitor, and evaluate personal progress [6].
This research investigates the effectiveness of WBTs and the relationship between students' SRL and their learning through WBTs.

\section{PROBLEM STATEMENT AND RESEARCH QUESTIONS}

Face-to-face class instruction can pose challenges to students. Statistics involves learning many complex concepts and procedures. In such cases, WBTs may be used as a tool, to provide outof-classroom instruction to enhance learning.

Web-based tutorials may pose problems associated with a lack of SRL skills. SRL skills include goal setting, self-monitoring, self-evaluation, use of learning strategies, help seeking, and time planning and management [7]. Learning through WBTs is student-centered in that students must practice self-regulatory skills to accomplish their learning goals [8]. It is expected that experienced students regulate their own learning skilfully. However, many often stick to high school or grade school learning strategies that prove to be insufficient to the college environment [9].

Online classes and web-based tutorials are part of distance education, however, they do have some differences. Online classes make use of synchronous/asynchronous communication tools like chat, email, and forums. Web-based tutorials require a shorter learning span, and do not normally have facilities where students can participate in synchronous/asynchronous communication.

While there is ample research on SRL, less research [10] has been done in relation to WBTs. Research is necessary to determine if WBTs are effective in students' comprehension of concepts and whether students' performance in WBT learning is related to their self-regulation strategies. 
The research on the effectiveness of WBTs shows that students are satisfied with learning through WBTs [11]-[19]. Belawati [20] found that students' participation in online tutorials improves course completion rates and achievement. Information gathered from this study will assist in the design of more WBTs.

Zimmerman [21] has shown that self-regulation is a reliable predictor of academic performance. According to Zimmerman [21], self-regulated learning theories of academic achievement are distinct from other means of learning due to two main reasons, how students select, organize, or create beneficial learning environments for themselves, and how they plan and control the form and amount of their own instructions. Zimmerman [21] has concluded in his overview study of SRL and academic achievement that systematic efforts can be launched to teach self-regulation to students who approach learning passively. According to Zimmerman [21], "A self-regulated learning perspective on students' learning and achievement is not only distinctive, but it has profound implications for the way teachers should interact with students and the manner in which schools should be organized. Accordingly, it is important to know the relationship between SRL and students' learning performance using WBTs.

The objective of the study is to seek answers to the following research questions:

1. Is a web-based tutorial effective in helping students understand difficult concepts in statistics?

2. Is there any difference between students' learning using WBT instruction and classroom instruction mode?

3. Is there any relationship between students' SRL and their WBT learning performance?

4. Are students' SRL independent of their learning style?

5. How satisfied are students with learning using WBTs?

Participation in this study will assist students with their awareness of their SRL strategies. Results of the study will provide insight to both students and teachers on how to improve and stimulate SRL strategies.

\section{III.LITERATURE REVIEW}

A large body of research exists on the effectiveness of learning and teaching through
WBTs. These studies compare online and face-toface learning approaches. Some of this research shows that WBTs are more effective than classroom instruction while others show that WBTs are as effective as classroom instruction. For example, researchers [22, 23, 24] found that web-based tutorials can accelerate the learning process with the same level of achievement as a classroom lecture. O'Neal, Jones, Miller, Campbell, and Pierce [25] showed that web based instruction is as effective as traditional teaching for disseminating special education course content to pre-service teachers. Fernandez [26] found no significant difference in learning through a classroom lecture and using a web-based tutorial. Similar results were found in a study by Nichols, Shaffer, and Shockey [27], which compared student learning through an online tutorial to a traditional lecture and found that students were satisfied with online instructions. Sweeney, O'Donoghue and Whitehead [28] suggested that a balance is needed between face-to-face and webbased tutorial learning approaches.

The effectiveness of WBTs has been investigated in almost every subject, chemistry [16], engineering [18], library sciences [17], forensic science [15], medical [14], and psychology [19]. These studies found that WBTs are as effective as classroom instruction.

Aberson, Berger, Emerson, and Romero [11, 12], and [13] explored the effectiveness of WBTs for statistics concepts. Aberson et al found that students were more satisfied with WBT learning therefore, attempts were made to improve the learning through the design of more WBTs.

Recent research related to SRL shows that SRL is one of the reliable factors that can be linked to personal and academic achievement of students.

SRL was validated by Usher and Pajares [29] in which Bandura's Children Self-Efficacy Scale was assessed using a sample of 3,760 students from grade 4 to 11 . The scale formed a one-dimensional construct and demonstrated an equivalent structure for boys and for girls, and for elementary, middle, and high school students. Thus, the scale provided a sound measure with which researchers can continue to assess students' beliefs about their self-regulatory capabilities.

Dabbagh and Kitsantas [8] point out that Webbased learning approaches are student-centered and web-based learning tools like emails, forums and chat can support students' development of selfregulatory skills that are essential for success in student-centered web-based learning environments. 
Mupinga, Nora, and Yaw [30] suggest that the design of online learning activities should strive to accommodate multiple learning styles. Garland and Martin [31] examined the differences between the learning styles of 168 students in online and traditional face to face courses and found a significant difference: "the learning style of the online student as a group was assimilating, while the learning style of the face-to-face student as a group was diverging". The authors concluded that the learning style and gender of all students must be considered when designing online courses.

\section{IV.METHODOLOGY}

Participants in this study consist of graduate students enrolled in a graduate research methods and statistics course at a Mid-western public university. Students were informed of the purpose of the study and completed an informed consent agreement.

This study used a single group, pre-test post-test, repeated measures, quasi-experimental design to (1) evaluate the effectiveness of web-based tutorials for learning statistical concepts using classroom teaching as a control group, and (2) to investigate the relationship between students' learning performance using WBT and their SRL.

Two pairs of related statistical concepts were selected $-z$ test/Chi square goodness of fit test and independent-groups/correlated-groups $t$ tests. WBTs were designed for two of these statistical concepts: $z$-test for single group and $t$ - test for independent groups, referred to as WBT-1 and WBT-2 respectively. The two WBTs can be viewed at http://publications.cit.iupui.edu/efernand/WBT1/ind ex.html

http://publications.cit.iupui.edu/efernand/WBT2/ind ex.html respectively. The other two concepts (Chisquare and t-test for correlated groups) were taught using classroom instruction. These two topics were used as a control group for the related experimental components.

Gagné and Briggs [32] have emphasized that in order to implement an effective learning process, it is important to evaluate students' understanding of the concepts as well as to get the feedback from students during evaluation. A pre-test was administered prior to the start of each concept mentioned above. The pre-tests for the $z$ test and Chi square were combined as were the pre-tests for the independent-groups and correlated-groups $t$ tests. After each concept's learning exposure, a post-test was administered. Fig. 1 provides a graphical representation of this procedure.
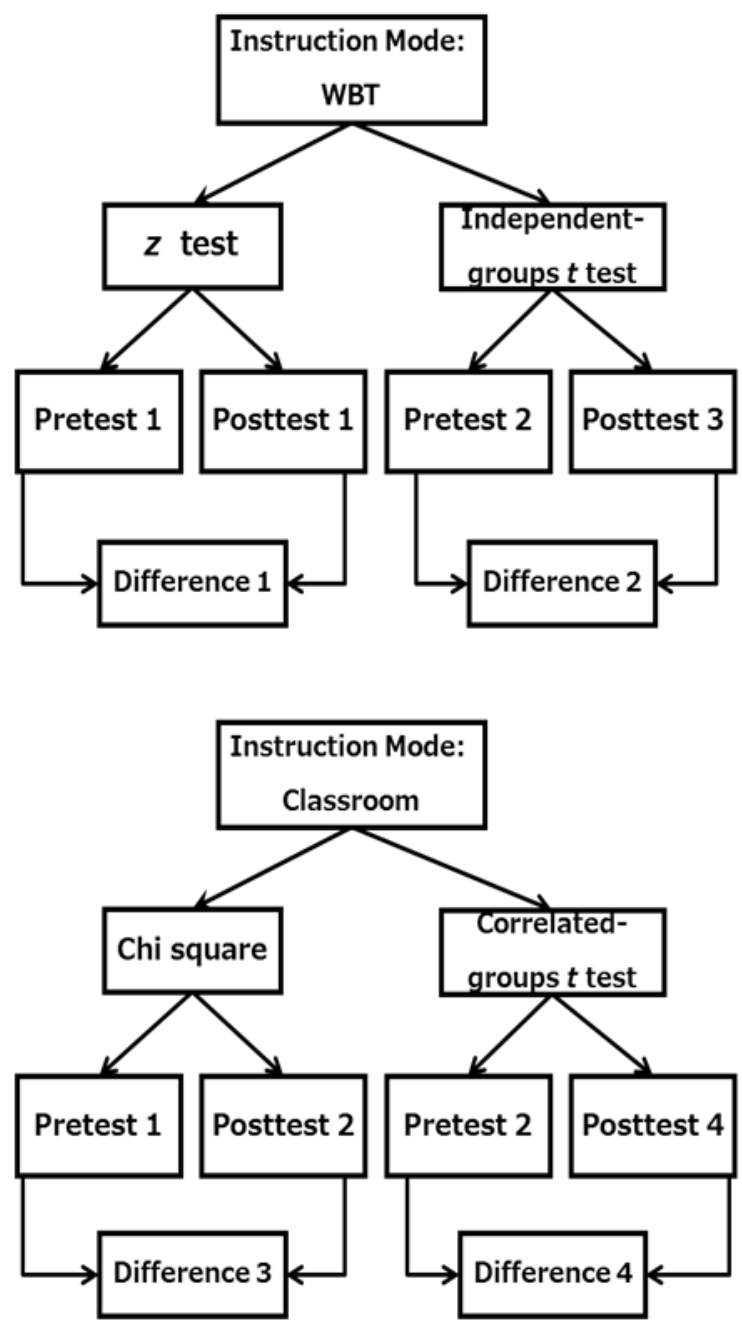

Fig. 1 Methodology

A difference score (post-test - pre-test) was then computed for each concept. Table 1 lists how each difference score was interpreted

Administering the online questionnaires, Keirsey Temperament Sorter II [34] allowed researchers to determine learning style. The learning style, demographic survey, and students' SRL scale were administered prior to the start of any experimental components. The students' self regulation strategies were evaluated using one subscale from the Children's Multidimensional Self-Efficacy Scales, namely self-efficacy for self- regulated learning. The 
scale measured students' perceived capability to use a variety of self-regulated learning strategies. Students' responses were recorded according to a 7point scale ranging from not well at all for a rating of 0 , not too well for 3 , pretty well for 5 , and very well for 7. Students' SRL was calculated by adding the score of 11 items for each students and then taking an average of that score, as has been done in other studies [35], [36]. As discussed in the literature review, the SRL scale was validated by Usher and Pajares [29].

Table 1: Use of difference scores

\begin{tabular}{|l|l|}
\hline Measure & Used to Evaluate \\
\hline Difference 1 & $\begin{array}{l}\text { Effectiveness of WBT on } z \text { test } \\
\text { independent-groups } t \text { test }\end{array}$ \\
\hline Difference 2 & $\begin{array}{l}\text { Effectiveness of classroom } \\
\text { instruction on Chi square } \\
\text { goodness of fit }\end{array}$ \\
\hline Difference 3 & $\begin{array}{l}\text { Effectiveness of classroom } \\
\text { instruction on correlated-groups } t \\
\text { test }\end{array}$ \\
\hline Difference 4 & $\begin{array}{l}\text { Effectiveness of WBT vs. } \\
\text { classroom instruction }\end{array}$ \\
\hline $\begin{array}{l}\text { Difference 1 - } \\
\text { Difference 3 and } \\
\text { Difference 4 }\end{array}$ & \multicolumn{2}{|l}{} \\
\hline
\end{tabular}

\section{RESULTS}

Of the 19 students enrolled in the course, $14(57 \%$ male, $43 \%$ female) usable responses were obtained. Students who participated in the study but didn't complete both pairs of pre-tests and posttests were excluded from the data analysis. 50\% of students were 25-34 years old followed by the age group of 45 and over. $36 \%$ of the participants were full time students while $64 \%$ were part time students.

A. Research Question 1: Is a WBT effective in helping students understand the concepts in statistics?

A paired-samples $t$ test was calculated to compare the mean pre-test score before the exposure to learning through WBT-1 to the mean post-test score after the WBT-1 learning. The mean on the pre-test was $24 \%$ ( $s d=11.87$ ), and the mean on the post-test was $67 \%(s d=23.60)$. A significant increase from pre-test to post-test was found $(t(8)=$ 5.768, $p<.001)$.

A paired samples $t$ test was calculated to compare the mean pre-test score before the exposure to the learning through WBT-2 to the mean post-test score after the WBT-2 learning. The mean on the pre-test was $10 \%$ ( $s d=20.69$ ), and the mean on the post-test was $65 \%(s d=18.57)$. A significant increase from pre-test to post-test was found $(t(8)=$ $6.805, p<.001)$.

\section{B. Research Question 2: Is there any difference} between students' increase in knowledge after WBT learning and classroom learning?

A paired-samples $t$ test was calculated to compare the mean change in knowledge after learning through WBT-1 to the mean change in knowledge after classroom instruction on Chi square. The mean change in knowledge after learning through WBT-1 was $46 \%$ ( $s d=21.26)$, and the mean change in knowledge after classroom instruction was $77 \%$ ( $s d=19.80)$. A significant difference was found $(t(7)=-3.037, p<.05)$. Students learned more after classroom instruction than using the WBT-1.

A paired-samples $t$ test was calculated to compare the mean of change in knowledge after learning through WBT-2 to the mean change in knowledge after classroom instruction. The mean change in knowledge after learning through WBT-2 was $45 \%$ ( $s d=31.38$ ), and the mean change in knowledge after classroom instruction was $65 \%$ ( $s d$ $=20.18)$. A significant difference was found $(t(10)$ $=-2.541, p<.05)$. Students learned more after classroom instruction than using the WBT-2.

C. Research Question 3: Is there any correlation between students' SRL and their WBT performance?

A Pearson correlation coefficient was calculated for the relationship between students' SRL and their WBT-1 performance. A moderate correlation that was not significant was found $(r(7)=.441, p>.05)$. Students' SRL was not strongly related to their WBT-1 performance.

A Pearson correlation coefficient was calculated for the relationship between students' SRL and their WBT-2 performance. A moderate correlation that was not significant was found $(r(9)=.027, p>.05)$. Students' SRL was not strongly related to their WBT-2 performance.

\section{Research Question 4: Are students' SRL} independent of their learning style?

Only 11 of the 14 students completed the Kiersey Temperament Sorter, with 8 of the 11 falling into the Guardian temperament. Because of this clustering, an ANOVA comparing students' SRL by temperament type was not possible. For reporting purposes the SRL scores were divided into three 
categories: high $(\mathrm{SRL}>4)$, medium $(\mathrm{SRL}=4)$ and low (SRL < 4). Table 2 shows the cross tabulation between SRL level and students' Keirsey temperament.

Table 2: Count of SRL by Temperament

\begin{tabular}{|c|c|c|c|c|c|}
\hline \multicolumn{2}{|c|}{} & \multicolumn{4}{|c|}{ Temperament } \\
\cline { 3 - 6 } \multicolumn{2}{|c|}{ SRL } & Guardian & Rational & Idealist & Total \\
\cline { 2 - 6 } & Med. & 1 & 0 & 0 & $\mathbf{1}$ \\
\hline & High & 8 & 1 & 1 & $\mathbf{1 0}$ \\
\hline \multicolumn{2}{|r|}{ Total } & $\mathbf{9}$ & $\mathbf{1}$ & $\mathbf{1}$ & $\mathbf{1 1}$ \\
\hline
\end{tabular}

E. Research Question 5: How satisfied are students with their change in knowledge using WBTs?

11 out of 14 participants responded to the satisfaction questionnaire. $45 \%$ of the students were 'somewhat satisfied' with WBTs while $36 \%$ were neutral about it. Two participants were dissatisfied with the tutorial. Satisfied students liked the content/information presented in the WBT while the dissatisfied students reported lack of interactive features and necessity of more illustrative examples. A total of $60 \%$ of the respondents said they would be 'likely' to study similar tutorials. None of the students reviewed any other resources on the topic taught using WBT-1 and WBT-2.

\section{CONCLUSIONS}

Clearly, there was a significant increase in students' gain in knowledge as a result of using the WBT. This result is consistent with the literature that shows WBTs are just as effective a learning medium as classroom instruction [14 - 19], [26]. More specifically, it confirms that WBTs were effective for learning statistics concepts, similar to studies by Aberson, Berger, Emerson, and Romero [11], [12] and [13]. However, our results were influenced by the uncontrollable confound of students reading the textbook chapter before the WBT exposure. 64\% $(7 / 11)$ and $67 \%(8 / 12)$ of students read/skimmed through the textbook chapter before they studied WBT-1 and WBT-2 respectively. Future researchers should make sure that WBTs are used as stand alone learning tools. An experimental study with a control group should be designed to make sure that WBTs are studied using an experimental design.

An examination of the learning differences between WBTs and classroom instruction showed that the classroom instruction was more effective than WBT instruction. This may be due to the fact that the pair of topics taught through WBTs and classroom instructions were comparable. In both situations, the WBT topic was introduced first and then the related topic was taught using classroom instruction. This design might have prepared the students' mindset first through the WBT and repetition may have helped them understand the second topic in the classroom setting more easily. Future studies should investigate the change in knowledge by reversing this sequence. Reversing the sequence would include teaching the concepts first and then using the WBTs to reinforce the material. However, coupled with the results of the first hypothesis this research validates the usefulness of WBTs as a supplemental method of instruction. Additional research could examine various types of hybrid courses to show if there is a significant increase in comprehension. Further research could include student's comprehension by age. Such as, do younger students tend to improve their knowledge more with WBTs versus face-to-face learning. Finally, research could include whether gender is a determining factor in the learning differences between WBTs and classroom instruction.

The results of the correlation test between SRL and WBT performance was interesting. In the present study, the majority of the students were between the ages of 25-34 and above the age of 45. Generally, students in these age group categories would be considered "experienced students." Experienced students should have exhibited high SRL scores. However, the WBT performance didn't indicate a proportional increase, demonstrating no correlation between SRL and WBT performance. Again, future research could examine the correlation between age and performance.

The lack of correlation between SRL and the WBT performance may be attributed to lack of motivation to learn using the WBT as the participants were enrolled in a face-to-face class. Some students reported that they didn't study the tutorial (27\% and 33\% students did not study WBT1 and WBT-2 respectively), which may indicate their lack of motivation to learn using WBT. and respond to related post-tests as compared to their class work. Some students reported that the WBTs lacked interactive features. In the future replication of such a study, it would be helpful to determine what interactive features are desirable and then design the WBTs accordingly. This could be accomplished by designing a mixed methods study that included both qualitative and quantitative data gathering. The qualitative data gathering could be 
focused on usability and user friendly aspects of various WBT's that had been created. The quantitative aspect of the study could measure the user satisfaction with various interactive features of the WBT's that were created as a result of feedback from the qualitative data gathering.

The sample size in the present study was small and the participants were graduate students who exhibited high SRL scores. Future researchers could increase the sample size of participants to show a stronger correlation or lack of correlation. It would be interesting to replicate this study with undergraduate students enrolled in traditional and online classes and give WBT learning treatment to both groups. Additionally, research could be done with similar groups of graduate students. This would allow researchers to see a correlation between graduate students SRL scores compared to undergraduate students with potentially lower SRL scores.

Student satisfaction with the WBTs was mild due to their desire for more interactive features and illustrative examples. This speaks to the high level of expectations on the part of the students for online materials. Thus, this research has shown that WBTs do have value and can be used as a supplement to classroom teaching, but they should be designed to include interaction.

\section{REFERENCES}

[1] G. V., Davidson-Shivers and K. L. Rasmussen. Webbased learning design, implementation and evaluation. N.J.: Prentice Hall, 2006.

[2] D. R. Forsyth and C. R. Archer. "Technologically assisted instruction and student, mastery, motivation, and matriculation." Teaching of Psychology, 24, 207212, 1997.

[3] V. A. Kazmerski and D. G. Blasko. "Teaching observational research in introductory psychology: Computerized and lecture-based methods." Teaching of Psychology, 26, 295-298, 1999.

[4] L. Liu, "Web-based resources and applications: Quality and influence." Computers in the Schools, 21(3/4), 131-47, 2004.

[5] T. P. Mackey and H. Jinwon, "Exploring the relationships between Web usability and students' perceived learning in Web-based multimedia (WBMM) tutorials." Computers \& Education, 50(1), 386-409, 2008.

[6] B. J. Zimmerman, "A social cognitive view of selfregulated academic learning." Journal of Educational Psychology, 81, 329-339, 1989.

[7] B. J. Zimmerman, "Investigating self-regulation and motivation: Historical background, methodological development, and future prospects." American Educational Research Journal, 45(1), 166-183, 2008.

[8] N. Dabbagh and A. Kitsantas, "Supporting selfregulation in a student-centered Web-based learning environments." International Journal of E-Learning, 40-47, January-March, 2004.

[9] B. K. Hofer, S. L. Yu, and P. R. Pintrinch, "Teaching college students to be self-regulated learners." In D. H. Schunk \& B. J. Zimmerman (Eds.), Self-regulated learning: From teaching to self-reflective practice (pp. 57-85). New York: Guilford, 1998

[10]P. M. Beile, and D. N. Boote, "Does the medium matter? A comparison of a web-based tutorial with face-to-face library instruction on education students' self efficacy levels and learning outcomes." Research Strategies, 20(1-2), 57-68, 2004.

[11]C. L. Aberson, D. E. Berger, E. P. Emerson, and V. L. Romero, "WISE: Web interface for statistics education." Behavior Research Methods, Instruments, \& Computers, 29(2), 217-221, 1997.

[12]C. L. Aberson, D. E. Berger, E. P. Emerson, and V. L. Romero, "Evaluation of an interactive tutorial for teaching hypothesis testing concepts." Teaching of Psychology, 30(1), 75-78, 2007.

[13]N. G. Bliwise, "Web-based tutorials for teaching introductory statistics." Journal of Educational Computing Research, 33(3), 309-325, 2005.

[14]P. R. Buzzell, V. M. Chamberlain, and S. J. Pintauro, "The effectiveness of web-based, multimedia tutorials for teaching methods of human body composition analysis." Advances in Physiology Education, 26, 2129, 2002.

[15]N. Daeid, "The development of interactive World Wide Web based teaching material in forensic science." British Journal of Educational Technology, 32(1), 105-108, 2001.

[16] W. Donovan and M. Nakhleh, "Student use of webbased tutorial materials and understanding of chemistry concepts." The Journal of Computers in Mathematics and Science Teaching, 26(4), 291-327, 2007.

[17]S. Michel, S. "What do they really think? Assessing student and faculty perspectives of a Web-based tutorial to library research." College \& Research Libraries, 62(4), 317-332, 2001.

[18]Z. Nedic and J. Machotka, "Interactive electronic tutorials and web based approach in engineering courses." Proceedings of the 5th IASTED international conference on Web-based education Puerto Vallarta, Mexico, 243-248, 2006.

[19]S. P. Wilson and A. Harris, "Evaluation of the Psychology Place: a Web-based instructional tool for psychology courses." Teaching of Psychology, 29(2), 165-168, 2002.

[20]T. Belawati, "The impact of online tutorials on course completion rates and student achievement." Learning, Media and Technology, 30(1), 15-25, 2005.

[21]B. J. Zimmerman, "Dimensions of academic selfregulation: A conceptual framework for education." In D. H. Schunk \& B. J. Zimmerman (Eds.), Self- 
regulation of learning and performance: Issues and educational applications (pp. 3-21). Hillside, NJ: Lawrence Erlbaum Associates, 2004.

[22]B. J. Zimmerman, "Self-regulated learning and Academic achievement: An overview." Educational Psychologist, 25(1), 3-17, 1990.

[23]C. Aivazids, M. Lazaridou, and G. F. Hellden, “A comparison between a traditional and an online environmental educational program." The Journal of Environmental Education, 34(4), 45-54, 2006.

[24]T. M. Day, M. R. Raven, and M. E. Newman, "The effects of World Wide Web instruction and traditional instruction and learning styles on achievement and change in student attitudes in a technical writing in an agricommunication course", Journal of Agricultural Education, 39(4), 65-75, 1998.

[25]K. O’Neal, W.P. Jones, S. P. Miller, S. P. Campbell, and T. Pierce, "Comparing Web-based to traditional instruction for teaching special education content." Teacher Education and Special Education, 30(1), 3441, 2007.

[26]E. Fernandez, "The effectiveness of Web-based tutorials", Proceedings of the Sixteenth International Conference on Technology and Education, Edinburgh, Scotland, March 28-31, 268-270, 1999.

[27]J. Nichols, B. Shaffer, and K. Shockey, "Changing the face of instruction: Is online or in-class more effective?" College \& Research Libraries, 64(5), 378388, 2003.

[28]J. Sweeney, T. O’Donoghue, and C. Whitehead, "Traditional face-to-face and web-based tutorials: A study of university students' perspectives on the roles of tutorial participants." Teaching in Higher Education, 9(3), 311-323, 2004.

[29]E. L. Usher, and F. Pajares, "Self-efficacy for selfregulated learning: A validation study", Educational and Psychological Measurement, 68(3), 443-463, 2006.

[30]D.M. Mupinga, R. T. Nora and D. C. Yaw, "The learning styles, expectations, and needs of online students", College Teaching, 54(1), 185-194, 2006.

[31]D. Garland, and B. N. Martin, B. N. "Do gender and learning style play a role in how online courses should be designed?" Journal of Interactive Online Learning, 4(2), 67-81, 2005.

[32]R.M. Gagné, and L. J. Briggs, Principles of Instructional Design ( $2^{\text {nd }}$ ed.). New York, NY: Holt, Rinehart and Winston, 1979.

[33]M. Riel and L. Harasim, "Research perspectives on network learning", Machine-Mediated Learning, 4(23), 91-113, 1994.

[34]D. Keirsey, Keirsey.com Retrieved July 22, 2008 from http://www.keirsey.com/, n.d.

[35]C. A. Carroll and L.S. Garavalia, "Gender and racial differences in select determinants of student success." American Journal of Pharmaceutical Education, 66, 382-387, 2002.

[36]M. Inzlicht, L. McKay and J. Aronson, "Stigma as ego depletion: How being the target of prejudice affects self-control." Association for Psychological Science 17(3), 262-269, 2006. 


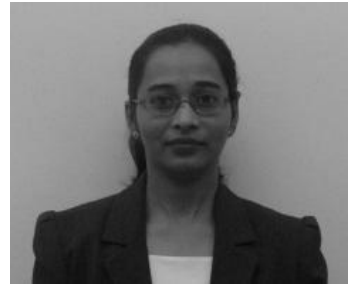

Swati Nere earned her B.E. (computer science and engineering) from $\mathrm{Dr}$. B.A.M. University in India and M.S. in Computer and Information Technology from the Purdue School of

Engineering and Technology at Indiana University Purdue University Indianapolis.

She is an Associate Software Engineer at Accenture, Chicago. She is also a visiting professor at DeVry University. She has over 5 years of teaching experience and her research interests include distance education systems and theories, teaching and learning in distance education, learning styles and instructional designs.

She can be contacted at swati.nere@gmail.com.

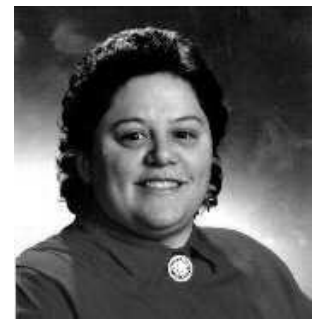

Eugenia Fernandez earned a B.S. in Mechanical Engineering from Worcester Polytechnic Institute, an M.S.E. in Computer, Information and Control Engineering from the

University of Michigan and a Ph.D. in Management Information Systems from Purdue University.

She is an Associate Professor of Computer and Information Technology and chair of the Department of Computer, Information \& Leadership Technology in the Purdue School of Engineering and Technology, Indiana University Purdue University Indianapolis. She is a fellow of the Mack Center at Indiana University for Inquiry on Teaching and Learning and an editor of the Journal of Scholarship of Teaching and Learning. Her research focuses on the scholarship of teaching and learning related to learning with technology.

Dr. Fernandez is a member of the Association for Computing Machinery (ACM), the Special Interest Group on Information Technology Education (SIGITE) and the American Society of Engineering Education (ASEE).

She can be contacted at efernand@iupui.edu.

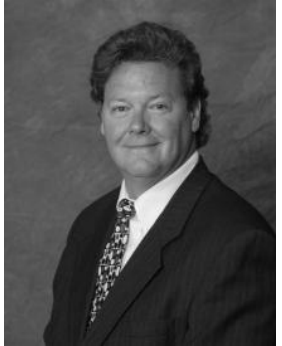

Charles Feldhaus, Ed.D. earned the B.A. in Radio and Television from the University of Southwestern Louisiana and the MS in Secondary Education from Indiana University. $\mathrm{He}$ earned his doctorate from the University of Louisville.

$\mathrm{He}$ is an Associate Professor of Organizational Leadership and Supervision and chair of Graduate Technology Programs with the Department of Computer, Information \& Leadership Technology in the Purdue School of Engineering and Technology, Indiana University Purdue University Indianapolis. His research focuses on the STEM Education, STEM Leadership and STEM Workforce Development.

He can be contacted at cfeldhaus@iupui.edu.

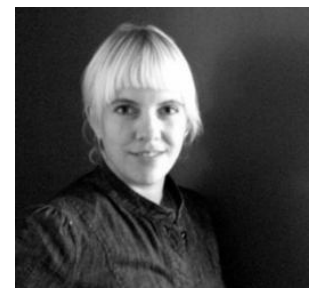

Jill Goodwin earned a B.S. in Informatics from Indiana University, and is pursuing a M.S. in Technology Purdue School of Engineering and Technology at Indiana University Purdue University Indianapolis.

She is a Graduate Research Assistant in Department of Computer, Information \& Leadership Technology in the Purdue School of Engineering and Technology, Indiana University Purdue University Indianapolis. Her research interests include adult teaching and learning, training and development systems and theories, and leadership practices.

She can be contacted at jmgoodwi@iupui.edu. 